\title{
Commentary
}

\section{Timer switch to convert suction apparatus for negative pressure wound therapy application}

ntermittent negative pressure therapy has proved its advantages. This presentation has sought to propose a cost effective Integrated Circuit (IC) based Electronic circuit to create breaking in Electric supply for specified time, and restoring it for specified time in cyclic manner automatically. The two ICs used in the equipment are Regulated IC 7812 and Timer IC 555. The IC 7812 is the voltage regulator IC which gives $+12 \mathrm{~V}$. Interesting to note the label, the 78 denotes (+ve) while 79 denotes (-ve) and the last two figures show the output voltage. In other words, IC 7812 is a regulated voltage source that powers the rest of the circuit in particular devices. These are used in several other electronic equipment like power supply of a cord less microphone and clap switch. However, in few of these equipment they have been getting heated up. On inquiry, the authors of this paper confirmed using their timer circuit for several hours continuously, without major heating as well as fuse blowing. The IC 555 has interesting working pattern. This chip allows the current

\begin{tabular}{|l|l|}
\hline \multicolumn{3}{|c|}{ Access this article online } \\
\hline Quick Response Code: & Website: \\
\hline & www.ijps.org \\
\cline { 2 - 2 } & Dol: \\
\hline
\end{tabular}

to flow through, while monitoring the voltage build up within the acceptable pre-set value, and would stop further flow of current when the voltage level exceeds the pre-set limit. Incidentally, it is the most economic part of entire circuit, and may cost as less as Indian rupee 10 . Possibility of using this circuit for longer times without burning out could have happened due to the usage of a step down transformer along with high resistors in the circuit, and that could prove for the proposed circuit to be very sturdy. It is suggested that the circuit be supplied to 20 different centres and can be used on the vacuum generators while noting the heat generation, continuous run time, and fuse blowing or circuit burning episodes. Congratulations to the authors for bringing out valuable information that can actually reduce the cost of negative pressure therapy.

\section{Atulkumar Shah}

MS, MCh, MPhil, LLM Consultant Cosmetic Surgeon 20 Om Park, Near Andhra Bank, Stadium, Akota, Vadodara, Gujarat 390020 E-mail: atulbda@gmail.com

How to cite this article: Shah A. Timer switch to convert suction apparatus for negative pressure wound therapy application. Indian J Plast Surg 2014;47:417.

Source of Support: Nil, Conflict of Interest: None declared. 